\title{
La biblioteca virtual de la Universidad de Guayaquil como agente de transformación: Retos y oportunidades
}

\author{
The virtual library of the University of Guayaquil as a \\ transformation agent: Challenges and opportunities
}

\author{
MSc, Carlota Mabel Cevallos Ronquillo ${ }^{1}$ \\ carlota.cevallosr@ug.edu.ec \\ MSc, Janeth Díaz Vera. Magister² \\ janeth.diazv@ug.edu.ec. \\ PHD, Roosevelt Barros Morales 3 \\ roosvelt.barrosm@ug.edu.ec
}

Recibido: 1/09/2018; Aceptado: 1/11/2018

\section{RESUMEN}

El avance de las bibliotecas universitarias (BU) es condicionado por el desarrollo de la sociedad de la información, en medio de una economía globalizada, donde las tecnologías de la información y comunicación (TIC) poseen un papel determinante. Aparecen novedosos sistemas de comunicación, generación y transmisión de la información; se masifica el acceso a la información y se crean nuevos soportes y medios de comunicación. Este trabajo tiene como propósito mostrar la evolución de la biblioteca de la Universidad de Guayaquil como una manera de democratizar el conocimiento y fortalecer la formación académica. Además, se realiza una panorámica sobre los retos que enfrenta y la necesidad de convertirla en centro para el aprendizaje y la investigación.

Palabras Clave: biblioteca virtual, educación superior, recursos, aprendizaje e investigación

\footnotetext{
${ }^{1}$ Universidad de Guayaquil, Guayaquil, Ecuador.

2 Universidad de Guayaquil, Guayaquil, Ecuador.

3 Universidad de Guayaquil, Guayaquil, Ecuador.
} 
자

No. 21, 31 de enero de 2019

ISSN impreso: 1390 - 6321

\begin{abstract}
The advancement of university libraries (BU) is conditioned by the development of the information society in the midst of a globalized economy, where information and communication technologies (ICT) have a determining role. New systems of communication, generation and transmission of information appear and mass access to information and new media and media are created. The purpose of this work is to show the evolution of the library of the University of Guayaquil as a way to democratize knowledge and strengthen academic education. In addition, an overview is made of the challenges it faces and the need to turn it into a center for learning and research.
\end{abstract}

Keywords: virtual library, higher education, learning and research resources

\title{
Introducción
}

La biblioteca de la Universidad de Guayaquil ocupa un lugar fundamental en esa institución, siendo la parte medular para la formación académica e investigativa de docentes y estudiantes. Constituye un espacio de estudio, de consulta, de encuentro, de intercambio, que enriquece la enseñanza y favorece la autonomía y responsabilidad de los estudiantes, docentes investigadores y comunidad en general en el proceso de enseñanza aprendizaje. La concepción que se tiene de la biblioteca tradicional era ofrecer documentos (libros, revistas, tesis, artículos, entre otros.) en soportes físicos a través de servicios de préstamos y consultas. Esas políticas limitaban de alguna manera con el acceso a los documentos por la falta de ejemplares o la existencia de ejemplares desactualizados. Sin embargo, junto al crecimiento de los recursos informáticos, la Internet y el descenso de los costos para adquirir esos medios y servicios relacionados, potenciaron en los últimos 20 años el diseño y la creación de las bibliotecas digitales (BD) es decir, se inició la automatización de las bibliotecas tradicionales, con un crecimiento sostenido y en constante evolución.

Para (López Guzmán, 2003): "Biblioteca virtual, es aquella que hace uso de la realidad virtual para mostrar una interfaz y emular un ambiente que sitúe al usuario dentro de una biblioteca tradicional. Hace uso de la más alta tecnología multimedia y puede guiar al usuario a través de diferentes sistemas para encontrar colecciones en diferentes sitios, conectados a través de sistemas de cómputo y telecomunicaciones".

La Universidad de Guayaquil, implementa el Software libre PMB como sistema de gestión en el año 2013, instalado en el servidor del MAEUG (Maestría de Administración de Empresas UG) y en el 2014 se instala en la página web www.ug.edu.ec, implementándose las especificaciones técnicas para el ingreso por parte del personal de cada una de las bibliotecas de la Universidad. Se trata de un sistema informático de gestión de bibliotecas SIGB en open source basado en un servidor HTTP, una base de datos y PHP. Es un software robusto, potente y con muchas opciones de configuración, lo que convierte en un programa ideal para cualquier tipo de organización. Incorpora módulos para catalogación, circulación, administración, publicaciones periódicas, adquisiciones, edición, tesauro, control de autoridades, online public Access catalog (OPAC) y comunicación, con el fin de facilitar la difusión selectiva de información (DSI). 


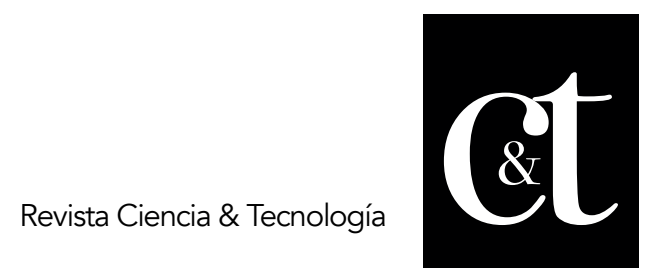

No. 21, 31 de enero de 2019

ISSN impreso: 1390 - 6321

Este artículo tiene como objetivo mostrar la evolución de la biblioteca de la Universidad de Guayaquil como una manera de democratizar el conocimiento y fortalecer la formación académica. Además, se realiza una panorámica sobre los retos que enfrenta y la necesidad de convertirla en centro para el aprendizaje y la investigación.

\section{Desarrollo}

La Biblioteca virtual fue pública en los años 90 Tiene como finalidad unir varias informaciones de diversos temas tales como artículos científicos, conferencias, actos académicos, libros, monografías, proyectos educativos y la unión se realiza gracias a la tecnología informática a través de una red operativa llamada internet. En la actualidad esta herramienta tecnológica es utilizada en los colegios, institutos superiores, universidades y en los diferentes ambientes educativos y porque no en los empresariales ya que se trata de datos informativos.

Para (Vega Belmonte, 2001): "Un conjunto de páginas web que, entrelazadas, en que todas ellas corresponden a una misma información o a una misma entidad. Es decir, el sitio web no es más que un conjunto de páginas web que tienen todas, como sistema, un objetivo común y están vinculadas e hipervinculadas"

Como su nombre lo indica, la biblioteca es un gran acopio estructurado de datos y conocimientos, con medios computarizados y cabida técnica asociadas para crear, buscar y usar la información. Abarca muchos datos, metadatos que explican varias características de la información -por ejemplo: autor, dueño de los derechos de publicación, etc.- y metadatos significa vinculación o relación con otros datos u otros metadatos, que pueden ser internos o externos a la biblioteca digital.

Biblioteca electrónica, es aquella que permite acceder a bancos de información en formato electrónico, cuenta con catálogos automatizados de bibliotecas tradicionales y los usuarios pueden reproducir la producción impresa, pero con un medio diferente del soporte papel. Cuenta con un espacio físico donde los usuarios pueden acceder a recursos impresos y digitales. La automatización del sistema de búsqueda de información es una de sus características. Realmente no es electrónica, correspondería a una biblioteca informatizada o automatizada.

"Es aquella que cuenta con sistemas de automatización que le permite una ágil y correcta administración de los materiales que resguarda, principalmente en papel. Así mismo, cuenta con sistemas de telecomunicaciones que le permiten acceder a su información, en formato electrónico, de manera remota o local. Proporciona principalmente catálogos y listas de las colecciones que se encuentran físicamente en un edificio. (López Guzmán, 2003)

La idea de implementar esta herramienta tecnológica en la Universidad de Guayaquil surgió por la demanda de libros y tesis de grandes cantidades de profesionales estudiantes que dejaban sus trabajos o proyectos de investigación en las bibliotecas para socializarlas o para consulta bibliográfica. Estas nuevas tendencias educativas 


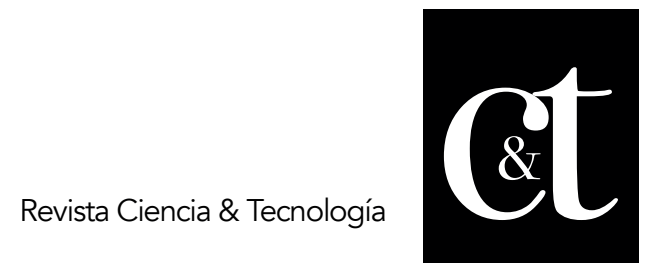

No. 21, 31 de enero de 2019

ISSN impreso: 1390 - 6321

son un nuevo esquema de ordenamiento de manera sistemática y cronológica que brinda a la población universitaria más comodidad, rapidez y efectividad en la información, esto permite que todas las facultades o sedes educativas, tengan una vinculación con la sociedad, lo cual es importante para fomentar la ciencia y la tecnología, como lo enfatizan Vega Belmonte (2003) y Adell (1997).

Biblioteca Virtual para (Zeni Marchiori, 1997): "La biblioteca virtual se conceptualiza como una especie de biblioteca que exista, que depende de la tecnología de realidad virtual". En este caso, un software propio conectado a un ordenador sofisticado reproduce el ambiente de una biblioteca en dos o tres dimensiones, creando un ambiente de inmersión total y la interacción. A continuación, es posible entrar en una biblioteca virtual, circular entre las habitaciones, seleccionar un libro en los estantes, "tocarlo", abrirlo y leerlo (Carrera, Delgado de los Santos, Contreras, 2017). La idea de implementar esta herramienta tecnológica en la Universidad de Guayaquil surgió por la demanda de libros y tesis de grandes cantidades de profesionales estudiantes que dejaban sus trabajos o proyectos de investigación en las bibliotecas para socializarlas o para consulta bibliográfica. Estas nuevas tendencias educativas son un nuevo esquema de ordenamiento de manera sistemática y cronológica que brinda a la población universitaria más comodidad, rapidez y efectividad en la información, esto permite que todas las facultades o sedes educativas, tengan una vinculación con la sociedad, lo cual es importante para fomentar la ciencia y la tecnología.

Obviamente, el único "lugar" donde realmente existe el libro está en la computadora y en la cabeza del lector" La meta de la Universidad de Guayaquil, es incrementar el número de visitas de estudiantes por medio del recurso tecnológico informático como es la biblioteca virtual, pero todo esto demanda para la institución de presupuesto, personal capacitado con conocimientos de software para la excelencia en operatividad. La institución se ha preocupado en la capacitación de los estudiantes creando la biblioteca virtual en la página web la que contiene libros, revistas, publicaciones, tesis, etc.

Para (Shmolling, 2001): "Una biblioteca virtual colecciona datos electrónicos, los ordena y los ofrece al lector; ella referencia informaciones diseminadas por todas partes del mundo."

Es necesario que el personal esté calificado profesionalmente, con competencias para clasificar, catalogar, indizar y ordenar la información, por lo que deberá estar constantemente actualizado con nuevos formatos, destrezas informáticas, enfocado al dominio y a las nuevas características, estar atento a la interacción entre las fuentes, servicios y captar las metodologías básicas en la búsqueda de información en ambiente electrónico, con preferencia a la privacidad y protegiendo los derechos de autor.

\section{Metodología}

Como parte de la metodología se recopiló la información necesaria para conocer las bases de datos digitales con las que cuenta la Universidad de Guayaquil, siendo estas adquiridas desde el año 2013, algunas de forma gratuitas y otras a través de contrataciones por convenios.

Como parte del proceso de acreditación, la contratación hasta el año 2013 se lo 
realizaba por medio de paquetes elaborados por la SENESCYT, para todas las universidades, pero en el año 2014 se pudieron adquirir en base a las necesidades institucionales de cada universidad.

La selección de base de datos se lo realizaba escogiendo bases multidisciplinarias que permitían el acceso a información contenida en libros, tesis, artículos científicos, revistas, para las diversas Facultades de la Universidad, como son las bases: Taylor \& Francis, Springer Ebook Journals, Gale Cengage, E. Technologies Proquest, ELibro E-brary, y Ebsco.

A continuación, se presenta los datos estadísticos que hacen referencia a los datos de las bases digitales de la Biblioteca Virtual de la Universidad de Guayaquil:

Tabla 1. Bases de datos contratadas a través de convenios en el año 20122013

\begin{tabular}{|l|r|}
\hline \multicolumn{1}{|c|}{ Base de datos } & \# de bases \\
\hline E. Tecnologies Proquest, E-libro e brary & 3 \\
\hline Taylor \& Francis & 4.391 \\
\hline Ebsco & 6 \\
\hline Springer & 5 \\
\hline Gale Cengage & 32 \\
\hline
\end{tabular}

Fuente: Archivo de la Biblioteca General de la Universidad de Guayaquil

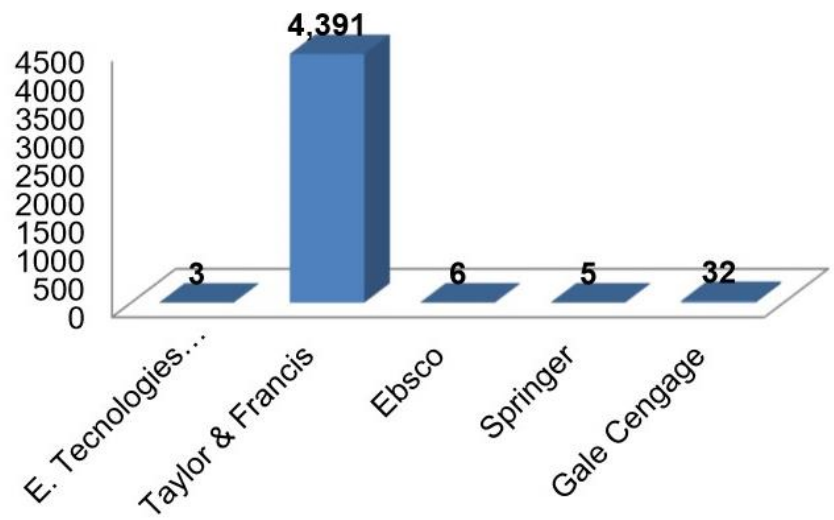

Gráfico 1. Bases de datos contratadas por convenio, 2012-2013

Fuente: Archivo de la Biblioteca General de la Universidad de Guayaquil

En el gráfico 1 se puede observar que de las bases de datos contratadas por la Universidad de Guayaquil en el año 2012-2013, a través de convenios la de mayor número de bases de datos es Taylor y Francis con 4.391 bases; seguido por Gale Cengage con 32 bases; Ebsco con 6; Springer 5; y E. Tecnologies Proquest, E-libro e brary con 3 bases de datos. Desde entonces se ha ido incrementando paulatinamente las bases de datos que alimentan la biblioteca virtual de la Universidad de Guayaquil. 


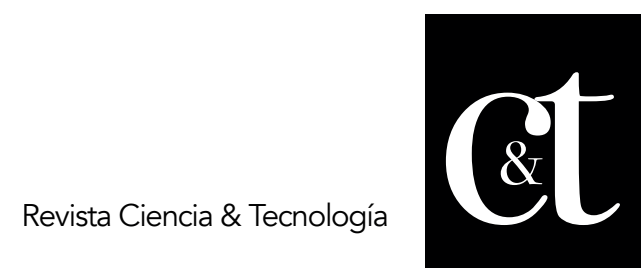

No. 21, 31 de enero de 2019

ISSN impreso: 1390 - 6321

Tabla 2. Presupuesto de las bases de datos adquiridas año 2012 y 2013

\begin{tabular}{|c|c|c|c|c|c|}
\hline \multirow{10}{*}{$\begin{array}{l}\text { Universidad } \\
\text { de Guayaquil }\end{array}$} & \multirow{5}{*}{2012} & \multirow{5}{*}{ Multidisciplinarias } & Cengage & $1,630.83$ & \multirow{10}{*}{$40,680.60$} \\
\hline & & & Ebsco & $3,029.79$ & \\
\hline & & & $\begin{array}{c}\text { E. Tecnologies } \\
\text { Proquest, E- } \\
\text { libro e brary }\end{array}$ & $5,546.67$ & \\
\hline & & & Springer & $4,796.88$ & \\
\hline & & & $\begin{array}{l}\text { Taylor \& } \\
\text { Francis }\end{array}$ & $3,446.81$ & \\
\hline & \multirow{5}{*}{2013} & \multirow{5}{*}{ Multidisciplinarias } & Cengage & $1,915.00$ & \\
\hline & & & Ebsco & $4,261.00$ & \\
\hline & & & $\begin{array}{c}\text { E. Tecnologies } \\
\text { Proquest, E- } \\
\text { libro e brary }\end{array}$ & $5,224.50$ & \\
\hline & & & Springer & $6,062.00$ & \\
\hline & & & $\begin{array}{l}\text { Taylor \& } \\
\text { Francis }\end{array}$ & $4,767.12$ & \\
\hline
\end{tabular}

Fuente: Universidad de Guayaquil 


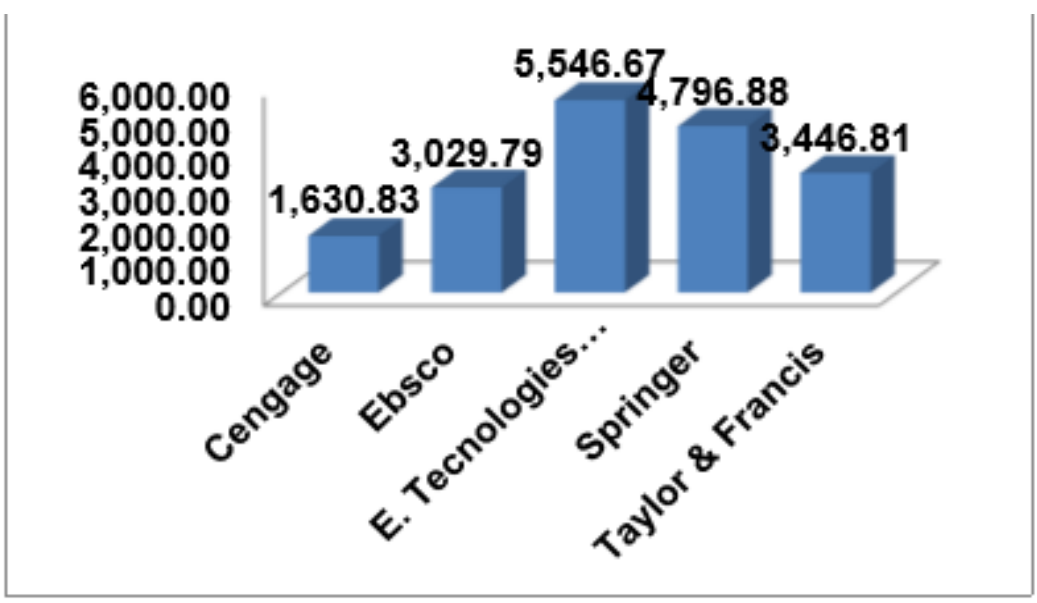

Gráfico 2. Presupuesto de las bases de datos, 2012

Fuente: Archivo de Biblioteca General de la Universidad de Guayaquil

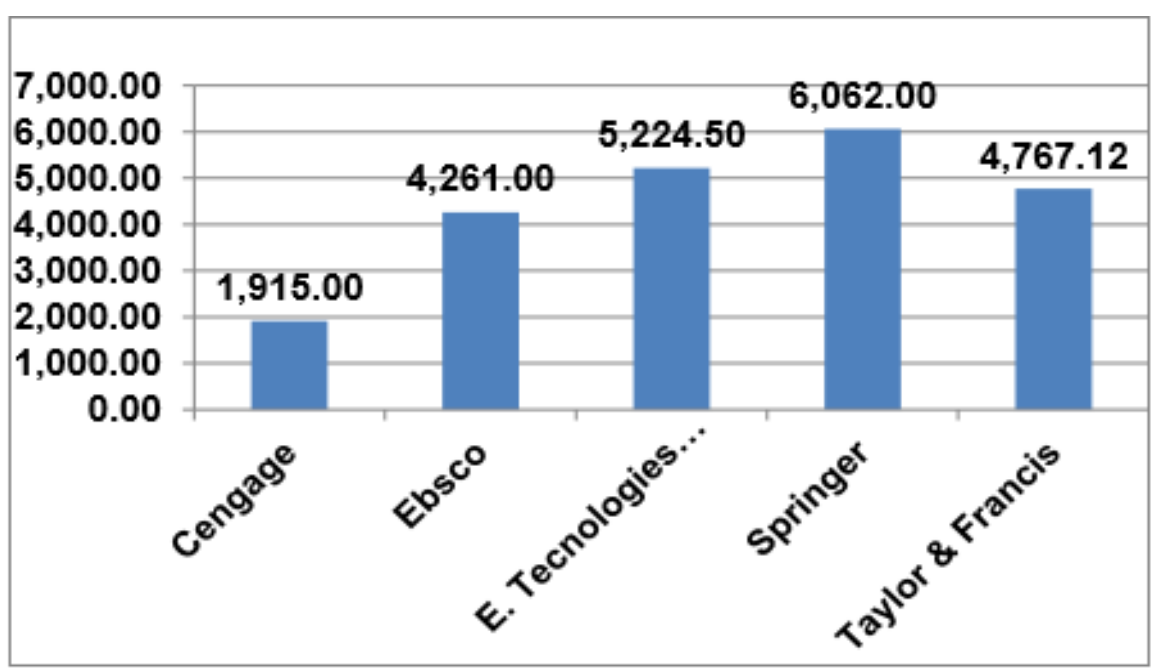

Gráfico 3. Presupuesto de las bases de datos, 2013

Fuente: Archivo de Biblioteca General de la Universidad de Guayaquil

Tabla 3. Bases de datos gratuitas, 2012 hasta la actualidad

\begin{tabular}{ll}
\hline \multicolumn{1}{c}{ Base de datos } & Contenido \\
\hline Flacso Andes & \\
BVS &
\end{tabular}

Fuente: Archivo de Biblioteca General de la Universidad de Guayaquil 
Tabla 4. Bases de datos contratadas a través de convenios en el año 2016

\begin{tabular}{|c|c|}
\hline Base de datos & Contenido \\
\hline Proquest & $\begin{array}{c}27 \text { bases de datos. } \\
\text { Más de } 19 \text {.ooo títulos de } \\
\text { publicaciones periódicas en } \\
\text { todas las áreas del } \\
\text { conocimiento } \\
\text { Más de } 50.000 \text { tesis } \\
\text { doctorales. } \\
\text { Paper } 23.601 \text { Artículos } \\
\text { científicos }\end{array}$ \\
\hline Springer & 6.000 libras electrónicas. \\
\hline e-libro & $\begin{array}{c}24.600 \text { libros electrónicos } \\
56.137 \text { libros en español }\end{array}$ \\
\hline Colección Alexander Street. & $\begin{array}{c}\text { Videos de apoyo académico } \\
\text { (5 temáticas) } \\
6.092 \text { videos }\end{array}$ \\
\hline Mc Graw Hill Educati & 60 libros electrónicas \\
\hline
\end{tabular}

Fuente: Archivo de la Biblioteca General de la Universidad de Guayaquil

Tabla 5. Presupuesto de las bases de datos adquiridas año 2016

\begin{tabular}{|c|c|c|c|c|c|}
\hline \multirow[t]{2}{*}{$\begin{array}{l}\text { Universidad } \\
\text { de Guayaquil }\end{array}$} & \multirow[t]{2}{*}{2016} & \multirow[t]{2}{*}{ Multidisciplinarias } & $\begin{array}{c}\text { Proquest, libros } \\
\text { electrónicos, } \\
\text { Ebrary y } \\
\text { E-libro } \\
\text { Cospivot }\end{array}$ & $27,100.43$ & \multirow[t]{2}{*}{$53,534.43$} \\
\hline & & & Libros virtuales & $26,434.00$ & \\
\hline
\end{tabular}

Fuente: Archivo de la Biblioteca General de la Universidad de Guayaquil

\section{Conclusiones}

Después de haber analizado la información referente a las bibliotecas de la Universidad de Guayaquil y los sistemas de recomendación que puede apreciarse que la integración de estos conceptos al contexto de bibliotecas digitales sugiere el desarrollo de sistemas que aumenten de una manera significativa la utilidad y la eficiencia de éstas.

La colaboración entre usuarios es la parte esencial en el proceso de búsqueda y agrupación de datos con diferentes bases de información y comunicación con accesos a la creación de grupos virtuales de usuarios que cooperan en el uso de bibliotecas digitales.

Se distingue, además, la importancia de la exploración de personas como fuentes de información.

Las aplicaciones futuras no deben olvidar que el desarrollo es en beneficio del usuario y por lo tanto ofrecerle herramientas que le ayuden a realizar su trabajo de una forma más eficiente. Para lograr esto es necesario considerar la forma natural en la que el 


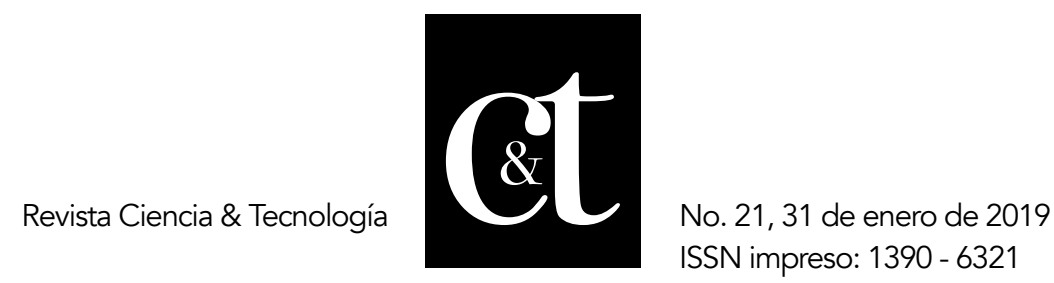

usuario interactúa con su ambiente.

Se recomienda fomentar a la Universidad de Guayaquil, convenios de información virtuales con software libre, y la adaptación de un sistema de repositorio digital compartido en las 17 facultades.

\section{Referencias bibliográficas}

Adell, J. (1997). Tendencias en educación en la sociedad de las tecnologías de la información. Revista electrónica de Tecnología Educativa. Vol. 7. Descargado de: http://www.edutec.es/revista/index.php/edutec-e/article/view/570/299.

Carrera, O., Delgado de los Santos, S. y Contreras, R. (2017). Factores que incentivan el uso de la biblioteca virtual en los estudiantes universitarios: Universidad de Gómez Palacios de Durango. Biblios. No. 66, pp. 98-111.

López Guzmán, C. (2003). Modelo para el desarrollo de bibliotecas digitales especializadas. Tesis de Posgrado. UNAM. México.

Shmolling, R. (2001). Las bibliotecas virtuales en Alemania: conecciones entre el saber, la información y la técnica. An Doc. No. 4, pp. 220-242.

Vega Belmonte, A. (2001). Web de calidad. La Habana, Cuba: Ed. Científico-Técnica.

Vega Belmonte, P. (2003). Aprenda web dinámico. La Habana, Cuba: Ed. CientíficoTécnica.

Zeni Marchiori, P. (1997). Ciberteca ou biblioteca virtual: una perspectiva de gerenciamiento de recursos de información. Ciencia da informacao. Vol. 26, No. 2, p.119. 